\title{
Rencontre avec Maurizio Bettini
}

(propos recueillis par Maria Paola Castiglioni)

Meeting with Maurizio Bettini (Interview by Maria Paola Castiglioni)

\section{OpenEdition}

\section{Journals}

Édition électronique

URL : https://journals.openedition.org/gaia/1301

DOI : $10.4000 /$ gaia. 1301

ISSN : 2275-4776

\section{Éditeur}

UGA Éditions/Université Grenoble Alpes

\section{Édition imprimée}

ISBN : 978-2-37747-199-7

ISSN : 1287-3349

\section{Référence électronique}

«Rencontre avec Maurizio Bettini », Gaia [En ligne], 22-23 | 2020, mis en ligne le 30 juin 2020, consulté le 09 décembre 2021. URL : http://journals.openedition.org/gaia/1301 ; DOI : https://doi.org/10.4000/ gaia.1301

Ce document a été généré automatiquement le 9 décembre 2021.

Gaia. Revue interdisciplinaire sur la Grèce archaïque 


\title{
Rencontre avec Maurizio Bettini
}

\author{
(propos recueillis par Maria Paola Castiglioni) \\ Meeting with Maurizio Bettini (Interview by Maria Paola Castiglioni)
}

\section{NOTE DE L'ÉDITEUR}

Maurizio Bettini est professeur de philologie grecque et latine et d'anthropologie du monde ancien à l'université de Sienne, où il a fondé, en 1986, le centre AMA (Antropologia e Mondo Antico), qui encourage la collaboration scientifique entre spécialistes de l'Antiquité classique et anthropologues, dans une approche pluridisciplinaire intégrant, dans une perspective anthropologique, les études historiques, archéologiques, philologiques et littéraires. Depuis 1992, il enseigne régulièrement au Department of Classics de l'University of California à Berkeley. Il est responsable de la collection « Mythologica » chez l'éditeur italien Einaudi et de la collection « Antropologia del Mondo Antico » chez Il Mulino. Il collabore en outre régulièrement avec le quotidien La Repubblica.

Parmi ses nombreux essais scientifiques, on signale : Antropologia e Cultura Romana (Rome, La Nuova Italia Scientifica, 1986) ; Nascere. Storie di donne, donnole, madri ed eroi, un lungo viaggio nel mondo della mitologia della nascita e delle credenze sugli animali (Turin, Einaudi, 1998); Voci. Antropologia sonora della cultura antica, studio antropologico della vocalità antica, umana e animale (Turin, Einaudi, 2008); Il ritratto dell'amante (Turin, Einaudi, 1992, traduit en français par G. Bouffartigue : Le portrait de l'amant(e), Paris, Belin, 2011) ; L'elogio del Politeismo. Quello che possiamo imparare oggi dalle religioni antiche (Bologne, Il Mulino, 2014, traduit en français par V. Pirenne-Delforge : Éloge du polythéisme. Ce que peuvent nous apprendre les religions antiques, Paris, Les Belles Lettres, 2016) ; Il grande racconto dei miti classici (Bologne, Il Mulino, 2015); Radici. Tradizione, identità, memoria (Bologne, Il Mulino, 2016, traduit en français par P. Vesperini : Contre les racines, Paris, Flammarion, 2017); A che servono i Greci e i Romani? (Turin, Einaudi, traduit en français par P. Vesperini : Superflu et indispensable. À quoi servent les Grecs et les Romains ?, Paris, Flammarion, 2018) et Homo sum. Essere «umani» nel mondo antico (Turin, Einaudi, 2019, traduit en français par M. Rousset Grenon : Contre les barbares, Paris, Flammarion 2020). On rappellera aussi la série de monographies consacrées à quelques 
figures emblématiques du mythe grec, coécrites avec d'autres spécialistes et publiées chez Einaudi : Il mito di Elena, avec Carlo Brillante (2002) (trad. française par

C. Pouzadoux, Le Mythe d'Hélène, Paris, Belin, 2010) ; Il mito di Narciso, avec Ezio

Pellizer (2003) (trad. française par J. Bouffartigue, Le Mythe de Narcisse, Paris, Belin, 2010) ; Il mito di Edipo, avec Giulio Guidorizzi (2004) (trad. française par V. Jolivet, Le Mythe d'Edipe, Paris, Belin, 2010); Il mito delle Sirene, avec Luigi Spina (2007) (trad. française par J. Bouffartigue, Le Mythe des sirènes, Paris, Belin, 2010) ; Il mito di Circe, avec Cristiana Franco (2010) ; Il mito di Enea, avec Mario Lentano (2013) ; Il mito di Arianna, avec Silvia Romani (2015) et Il mito di Medea, avec Giuseppe Pucci (2017).

Il est également auteur de romans de fiction.

Nous signalons aussi un volume récent (Dai Romani a noi, Bologne, Il Mulino, 2019), transcription d'une longue et passionnante conversation que Francesca Prescendi et Daniele Morresi ont eue avec Maurizio Bettini et dans laquelle est retracé le parcours scientifique de Maurizio Bettini et sont reprises les thématiques scientifiques qui ont jalonné sa carrière et les activités du centre AMA.

MAURIZIO BETTINI, JE VOUS REMERCIE TOUT D'ABORD POUR LE TEMPS QUE VOUS NOUS CONSACREZ. JE SUIS TRÈS HEUREUSE D'OUVRIR AVEC VOUS CETTE NOUVELLE RUBRIQUE DE GAIA, dédIÉE À des ReNCONTRES AVEC DES SPÉcIALISTES dU MONDE ANCIEN. PUISQUE

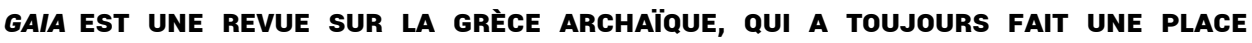
D'HONNEUR À HOMĖre, Je VOUdRAIS TOUT D'ABORd VOUS DEMANDER QUEL EST, SELON VOUS, LE SENS de L'ÉTUdE dE CE POÈTE AUJOURD'HUI ?

Homère est le fondement de la culture grecque. Il est rare, par exemple, qu'un mythe grec n'ait pas au moins deux vers dans les poèmes homériques. On y retrouve aussi beaucoup d'autres aspects de la culture grecque. Il est donc en ce sens fondamental. À cela s'ajoute la beauté, inatteignable, de ces deux poèmes, notamment de l'odyssée. Un autre élément qui me semble important est le sens d'altérité qui se dégage de la lecture d'Homère. Ce poète donne même concrètement le sentiment de la différence dans sa façon d'écrire, son style formulaire, ses répétitions, ses variations sur ce qu'il a déjà dit, ses retours. Il s'agit d'une façon d'écrire qui a été ensuite perdue et qui n'est pas, n'en déplaise à Parry et Lord, une simple technique, mais aussi un choix stylistique qui produit un texte unique, qui ne ressemble à aucun autre, car personne n'écrit ou parle ainsi. Cette construction poétique formulaire donne une dimension mythique au langage. C'est un peu ce qui se produira plus tard à Rome avec le style analogique qui, par exemple, itère les sons. Il s'agit d'un moyen de donner un caractère immédiatement concret au langage.

Cette écriture merveilleuse constitue déjà en soi une excellente raison pour lire Homère. Une autre raison qui rend ces poèmes si fascinants est la présence des dieux : dans les poèmes homériques, nous pouvons entrer dans le monde des dieux, « voir » ce qu'ils font, comment ils parlent, se disputent, raisonnent... Il s'agit d'une chance qui est offerte au lecteur, qui a par ailleurs le privilège de savoir comment ils apparaissent, comment ils sont faits, alors que les personnages de l'Iliade et de l'Odyssée ne voient jamais les divinités : elles apparaissent " voilées ", déguisées. C'est un paradoxe: même les grands héros n'en profitent pas, à l'exception de Diomède auquel Athéna enlève exceptionnellement et provisoirement, au chant $\mathrm{V}$ de l'Iliade, l'achlus des yeux, ce "brouillard », cette « brume » qui voile le regard des hommes et les empêche de jouir de la vue des dieux.

NOUS SOMMES dONC des PRIVILÉgIÉs gRÂCe À HOMÈRE, QUI EST EN QUELQUE SORTE UNe porte d'entrée remarquable vers le monde de la civilisation et de la langue 
grecques. l'enseignement de cette langue, tout comme celui du latin, est CEPENDANT AUJOURd'HUI de PLUS EN PLUS MENACÉ ET LES ÉtUdiantS EUX-MÊMES ONT PARFOIS dU MAL À EN AIMER L'APPRENTISSAge, EN JUgEANT LES MÉtHOdes POUSSIÉREUSES ET LES OBJECTIFS STÉRILES. VOUS AVEZ RÉCEMMENT POINTÉ DU DOIgT CE PROBLÈME dANS UN LIVRE INTITULÉ A CHE SERVONO I GRECI E I ROMANI? (TURIN, EINAUDI, TRADUIT PAR P. VESPERINI : SUPERFLU ET INDISPENSABLE. À QUOI SERVENT LES GRECS ET LES ROMAINS ?, PARIS, FLAMMARION, 2018) QUI EST AUSSI UN PLAIDOYER POUR L'UTILITÉ DE L'Étude des sciences de L'ANTIQUité POUR CoMprendre le PRÉsent et LA LONgUe TRADITION CULTURELLe QUi EN EST À L'ORIgINe, À CONDITION DE CHANgER LeS RÈgLeS DE

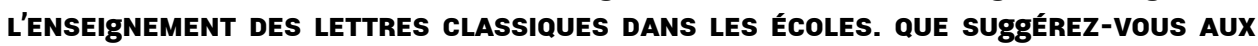
ENSEIgNANTS ET AUX ENSEIgNANTS-CHERCHEURS AfIN D'ENCOURAgER NOS ÉLÈVES ET NOS ÉTUDIANTS À ABORDER CETTE DISCIPLINE ?

Confrontés à la seule étude des déclinaisons, les étudiants ont du mal à cerner l'utilité de cet effort important qu'on leur demande. Une solution serait de partir d'un aspect de la culture et de la littérature antique, par exemple en lisant un passage d'un poème homérique ou d'une tragédie dans une traduction moderne et accessible et, une fois suscités l'intérêt et la curiosité des étudiants, de mettre en relation un mot grec significatif avec ce que le passage a permis d'étudier. En partant d'un élément lexical, comme le mot mythos avec ses multiples significations, il serait alors possible de conduire les étudiants vers une connaissance plus approfondie, qui passe nécessairement par l'initiation aux mécanismes de fonctionnement de la langue et à son vocabulaire. Mais la fatigue de l'apprentissage serait ainsi juste une étape pour assouvir un intérêt plus large, celui de la connaissance d'un monde qui apparaît au début aux étudiants comme différent, étrange, autre.

CET ASPECt de LA DIVERSITÉ EST JUSTEMENT Un ÉLÉMENT QUE NOUS, LES ENSEIgNANTS,

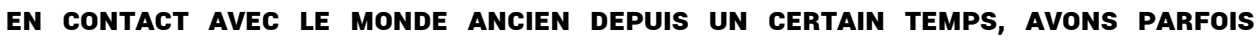
TENDANCE À OUBLIER...

Oui, mais le monde ancien est bien un monde différent et cet aspect doit être exploité car il suscite de l'intérêt. On peut par exemple montrer aux étudiants que les Anciens adorent une multiplicité de divinités et pas une seule et qu'ils honorent aussi les divinités des autres, les prennent très au sérieux, peuvent même se les échanger, peuvent identifier Zeus avec Jupiter et Mercure avec Odin. Cet aspect peut être mis en comparaison avec le fonctionnement des religions monothéistes actuelles, l'islam ou le christianisme. La différence est flagrante. De même, la distance est sensible entre notre société et les sociétés antiques qui exploitaient le travail des esclaves. Ces aspects peuvent être des éléments de réflexion et de questionnement: le monde antique acceptait l'esclavage, alors que la nôtre l'interdit au nom des droits de l'homme. On peut alors se demander comment les Anciens se posaient, eux, la question de droits de l'homme et de leur violation ${ }^{1}$. Il faudrait donc entraîner les étudiants dans une conversation qui est à la fois sérieuse et passionnante, sans se focaliser, se figer sur le seul apprentissage de la grammaire.

CETTE OUVERTURe À UNe COMPARAISON ENTRE ÉPOQUES DIFFÉRENTES M'ÉVOQUe UN AUTRE dÉSIR QUE MES ÉtUdiANTS d'HISTOIRE ONT À PLUSIEURS FOIS MANIFESTÉ, CELUI DE DÉCloIsONNER L'HISTOIRe gRECQUe et L'HISTOIRe ROMAINE, DONT LES ÉVÉNEMENTS CONTEMPORAINS SONT TROP SOUVENT PRÉSENTÉS COMME APPARTENANT À DEUX UNIVERS séparés, par allleurs trop souvent limités À ATHÈnes et rome, sans un ÉLARgISSEMENT AUX AUTRES ESPACES ET CULTURES DU MONDE ANCIEN...

Cela est très intelligent de leur part, car cette approche permet aussi quelque chose de fondamental dans nos études : la comparaison, qui est l'âme de la compréhension. Comme le disait Alexis de Tocqueville, «c'est une des infirmités singulières de notre 
esprit de ne pouvoir juger les objets, les vitt-ils en plein soleil, s'il ne place un autre objet à côté ». Cela est très vrai. Mises à côtés les unes des autres, les choses s'éclairent entre elles. Ainsi, ce qu'on n'arrive pas à voir de la Grèce du ve siècle av. J.C. peut être mieux compris à la lumière de ce qui se passe dans le monde romain au même moment. Cette comparaison n'est pas seulement recherche d'analogies, mais est aussi le point de départ afin de mieux saisir les différences. Par exemple, en revenant à la religion, si Hérodote affirme que ce sont les plus anciens des poètes, Homère et Hésiode, qui ont, par leurs poèmes, «fabriqué une théogonie, donné aux dieux leurs titres, réparti entre eux honneurs et compétences et fait connaître leurs formes ", Varron considère quant à lui que ce sont les institutions qui ont fait connaître les cultes. On voit ainsi deux mondes très différents : l'un, le grec, où le poète est aussi «théologien » et écrit la vie des dieux, et l'autre, le romain, avec ses règles dictées par les «institutions » de la ville, ses listes où on définit quels sont les jours destinés au culte de chaque divinité. À partir de là on peut mettre en comparaison ces mondes, et réfléchir aussi aux ressemblances qu'ils ont avec les religions actuelles.

Au-delà de la religion, il y a d'autres thèmes qui peuvent être étudiés et comparés, par exemple les institutions, les formes de gouvernement, ou, dans le cadre de l'histoire sociale, les rapports de genre ou encore la question de l'esclavage, ce dernier aspect étant un peu trop négligé. Cette organisation sociale explique aussi le fait que les sociétés anciennes, la société romaine notamment, étaient peu « racistes ", dans le sens où elles ne créaient pas d'oppositions radicales sur la base de la couleur de la peau ou des traits somatiques. Le vrai clivage était entre libres et esclaves. En simplifiant beaucoup, on pourrait dire qu'une fois perdue cette différenciation, la hiérarchisation s'est déplacée sur les traits somatiques, alors qu'auparavant l'esclavage touchait indifféremment les gens du Nord et du Sud, en identifiant l'esclave avec le «noir». Le fait que les Romains ne classaient pas les hommes sur la base de la couleur de la peau n'est pas simplement une vertu positive de leur ouverture - même si dès le départ la société romaine fut une société ouverte -, mais une conséquence de la division entre libres et non libres.

Encore une fois, tous ces thèmes, s'ils sont présentés dans un processus de découverte et de réflexion, apportent une motivation importante à l'apprentissage des langues anciennes, qui deviennent un moyen, un instrument, pour approfondir ce parcours. Il faut donner un sens à cet apprentissage. Je ne crois pas au charme de l'aoriste...

\section{UN AUTRE MOTEUR DE CURIOSITÉ IMPORTANT EST LE MYTHE...}

Il s'agit sans aucun doute de l'un des aspects de la culture ancienne les plus aimés et les plus connus. Je me rappelle qu'il y a quelques années j'animais une émission radiophonique intitulée C'era una volta il mito («Il était une fois le mythe») dans laquelle je parlais des mythes classiques. Le matériel de l'émission est ensuite devenu un petit livre, dans un deuxième temps augmenté et illustré. Ce livre a connu plusieurs impressions et, à ma grande surprise, est devenu, parmi mes livres, celui qui a eu le plus de succès ${ }^{2}$. Le mythe exerce encore un grand attrait.

Cet engouement pour le mythe classique se manifeste par allleurs par sa Récupération, entre autres, par les comics, la bande dessinée américaine, QUi ONT CRÉÉ À PARTIR des ANNÉEs 1940 des PERSONNAgES INSPIRÉs diRECTEMENT de Ce PATRIMOINE (L'AMAZONE WONDER WOMAN, PAR EXEMPLE, OU, POUR LE MYTHE NORDIQUE, THOR, fILS D'ODIN) ET DONT LES ADAPTATIONS CINÉMATOgRAPHIQUES CONNAISSAIENT UN 
SUCCÈs INÉgALÉ SUR LE gRANd ÉCRAN... LE MYTHE EST DONC INTEMPOREL. POURQUOI ? ET QU'EST-CE QU'IL APPORTE À L'HUMANITÉ d'AUJOURD'HUI, SI DIfFÉRENTE de CELLE dU PASSÉ ?

Comme le dit un peu paradoxalement Edgar Morin, nous descendons certes de l'homo sapiens, mais dans celui-ci il y a également l'homo demens, car il faut tenir compte aussi de la folie. De la même façon, il y a certes l'homo faber, mais aussi l'homo mythologicus, l'homme de la création fantastique. Le mythe est selon moi la représentation de cet homo mythologicus, l'homme qui est capable d'inventer des récits, des mondes qui n'existent pas. Il le sait et les crée justement pour ça. L'homo mythologicus est celui qui, au milieu des homines fabri, pris par leur rationalité et leur activité de production, par l'urgence de ce qui sert dans l'immédiat, donne naissance au mythe, quelque chose qui n'a pas d'utilité pratique instantanée, mais façonne les frontières de l'imaginaire. Le mythe grec - et c'est cela qui le rend si fascinant n'est jamais fini et son contenu n'appartient à personne. Le mythe est malléable et susceptible d'être enrichi - et il est tout à fait légitime de le faire. Aucune autre production fantastique ne permet cet élargissement à l'infini.

\section{LE MYTHE RÉPONd dONC À UN BESOIN DE L'HOMME? ET CELA EXPLIQUE-T-IL SON CARACTÈRE UNIVERSEL ET SON SUCCÈS INTEMPOREL?}

Oui, tout à fait. C'est la puissance de ce type de récits que nous appelons " mythes ", ces récits manifestement incroyables que personne ne prétend être vrais, mais qui sont importants en tant que tels. Ils fascinent, font penser... Il n'est pas facile d'écrire des mythes, bien entendu, et dans cela les Grecs se sont montrés maîtres en créant un univers.

\section{QUI NOUS PARLE ENCORE AUJOURD'HUI...}

Oui, car il s'agit de récits fantastiques mais liés à des grands thèmes de la culture : l'amour, les rapports conjugaux, l'inceste, le meurtre, la peine et la purification, l'aventure, la découverte... Pensons par exemple au mythe d'Amphitryon dans lequel Zeus est capable de prendre l'aspect de ce général thébain pour s'accoupler avec sa femme. Cela touche directement à la question de l'identité. C'est une invention grecque.

dANS CE SENS ON COMPRENd AUSSI L'INTÉRÊt de LA PSYCHANALYSE POUR LE MYTHE gREC.

Oui. Cet intérêt naît d'une heureuse rencontre, dans la Vienne de la fin du XIX ${ }^{\mathrm{e}}$ siècle, entre le mythe et les structures du profond grâce à des médecins qui avaient une excellente culture classique et étaient passionnés de mythe et de folklore. Cette association ne va pas du tout de soi. Personne n'y avait pensé avant eux. Cela a permis de produire une nouvelle signification du mythe qui a eu à son tour beaucoup de succès. Je me rappelle qu'il y a quelques années je donnais un cours sur la mythologie à Berkeley à une classe undergraduate, avec des étudiants non spécialistes formés à des disciplines scientifiques. Au début du cours, je leur demandai : « Qu'estce qu'est pour vous la mythologie? » L'un d'eux me répondit : «La mythologie est quelque chose qui concerne la psychologie des hommes et qui sert à comprendre le comportement. » Surpris, je lui demandai où il l'avait lu. Il me répliqua qu'on le lui avait enseigné à l'école. Cela montre à quel point le freudisme américain s'était approprié les mythes grecs, alors que pour nous la psychanalyse est seulement l'une des façons de réfléchir sur le mythe. 
Ces déclinaisons du mythe grec montrent alors Que Ce patrimoine deVRait Être UNE CONNAISSANCE COMMUNE, ACCESSIBLE À TOUS.

Tout à fait. Il ne s'agit pas d'une connaissance qui doit être réservée à une élite qui se reproduit en ne fabriquant que des antiquisants. Cela n'aurait aucun sens. Le lycée classique italien, par exemple, ne doit pas produire que des antiquisants, car sinon il aurait raté sa vocation. Il devrait en revanche former des individus cultivés grâce à une formation historique, philosophique, scientifique et aussi classique qui leur permette d'être à l'aise avec le passé classique et sa récupération tout en exerçant des métiers qui n'ont rien à voir avec le monde ancien.

\section{IL FAUDRAIT PEUT-ÊTRE ASSURER UNE FORMATION DE QUALITÉ SANS ÉLITISME...}

Oui, il s'agit d'un grand malentendu, entretenu aussi par une partie de ceux qui exercent des métiers dans le domaine de la culture et qui restent attachés à conserver cette distance, à ne pas se faire comprendre. La clarté devrait être une obligation morale. Il faut se faire comprendre et donner à son interlocuteur toutes les cartes pour débattre, même pour qu'il nous donne tort. Il est immoral de camoufler le savoir avec des discours incompréhensibles, souvent construits sur le vide.

en parlant de responsabilité, J'en profite alors pour vous demander quel DEVRAIT ÊTRE LE RôLE dU SPÉCIALISTE de L'ANTIQUITÉ dANS NOTRE SOCIÉTÉ ? JE PENSE À CERTAINS DE VOS LIBRES PLUS ENgAgÉS, ET NOTAMMENT À CONTRE LES BARBARES.

Ce rôle passe dans mon cas à travers l'anthropologie du monde ancien, discipline qui permet de sélectionner des thèmes de l'Antiquité classique qui entrent en résonance immédiate avec la contemporanéité. Bien entendu, l'engagement d'un traditional scholar, comme les appellent les Américains, peut se faire à titre personnel, indépendamment des sujets de recherche. La dimension de réflexion anthropologique permet cependant une confrontation avec les problèmes actuels beaucoup plus poussée que, par exemple, la simple analyse intertextuelle. Cela dit, la production d'un auteur ancien peut être le moyen de se confronter à des problèmes plus vastes. Par exemple, Venance Fortunat peut être étudié dans le cadre d'une réflexion plus large sur l'articulation entre culture romaine et barbare. Venance Fortunat cherche un langage commun entre deux cultures différentes qui sont entrées en conflit. Cette approche peut devenir un moyen utile de raisonner sur le présent. On peut également faire l'exemple de Rutilius Namatianus, poète qui raconte, dans De reditu suo, son voyage de Rome à Marseille : lors d'une des étapes de son voyage, il rencontre une communauté de Juifs qu'il décrit avec des affirmations sauvagement antisémites. Cela peut nous entraîner à nous interroger sur les racines d'un phénomène millénaire qui malheureusement perdure encore de nos jours et à se demander quelles en étaient les causes à l'époque romaine.

Si l'antiquisant veut avoir un rôle dans la société contemporaine, il faudrait qu'il choisisse des thèmes qui peuvent entrer en rapport avec celle-ci, et qu'il ait le regard de l'anthropologue, du philosophe ou de l'historien. Ce dernier a en ce sens une grande liberté de regard critique sur le passé et ne peut pas ne pas être engagé. Le sens de responsabilité civile vient avant le choix du sujet d'étude. Et l'enseignant a à ce propos un rôle privilégié. Tant que cette liberté dure...

nos disciplines sont en effet de plus en plus menacées. y A-t-IL Un Risque Réel D'EXTINCTION?

Si la menace existe, il faut aussi dire qu'au moins en Italie il y a un public prêt à soutenir ces disciplines, comme le montre le succès, dans les derniers quatre-cinq 
ans, des livres qui promouvaient la défense du grec et du latin. Pour éviter que la culture antique ne soit menacée d'extinction il faut d'une part s'investir dans le domaine de l'enseignement afin de susciter, comme on l'a dit, une passion pour ces disciplines auprès des étudiants, d'autre part innover au niveau universitaire, dans la formation des futurs enseignants, afin d'éviter de reproduire tout bêtement les mêmes contenus figés appris par nos parents et grands-parents, complètement décalés par rapport au monde contemporain et aux attentes des étudiants.

\section{QUELLES SERAIENT DONC LES NOUVELLES FRONTIÈRES DANS L'ÉTUDE DE L'ANTIQUITÉ ?}

La nouvelle frontière serait le dépassement d'une dimension purement littéraire de l'Antiquité, ainsi qu'une approche historique qui ne soit pas seulement une étude des institutions et des dates, mais aussi de la société et de la culture. Il s'agit d'un processus en partie amorcé il y a des décennies et qui se développe de plus en plus. Il faut sortir des «prisons" traditionnelles que sont l'étude des manuels de littérature et d'histoire afin de s'aventurer dans la mer ouverte de la culture ancienne. Cela se joue sur deux fronts : celui de l'altérité, car les mondes anciens sont différents et multiples, et il faut les explorer et les redécouvrir en tant que tels, mais aussi celui de l'identité, car ils constituent aussi notre passé millénaire, sur lequel s'est construite notre culture. Sans tomber dans le piège de l'actualisation des classiques, nous pouvons exploiter positivement cette proximité, même linguistique. Proust écrivait, au sujet du français, que « ces mots français que nous sommes si fiers de prononcer exactement ne sont eux-mêmes que des "cuirs" faits par des bouches gauloises qui prononçaient de travers le latin ou le saxon, notre langue n'étant que la prononciation défectueuse de quelques autres ». La lecture des poèmes homériques constitue un point commun entre Alexandre le Grand et nous, par exemple. Cette continuité est une porte qui permet d'entrer plus facilement en contact avec ces cultures. Ce "double visage » de l'Antiquité permet à la fois de comprendre plus facilement les tableaux du Louvre avec des sujets antiques, mais aussi de prendre conscience qu'il s'agissait d'une société qui pratiquait l'esclavage, qui marginalisait les femmes, qui pratiquait la pédérastie...

\section{LE DIALOgUE PLURIDISCIPLINAIRE POURRAIT AUSSI AIDER À RENOUVELER CE REgARD...}

Le dialogue avec d'autres disciplines, d'autres littératures est fondamental. Étudier un peu de sociologie ou d'anthropologie fait du bien à l'historien, tout comme la linguistique peut être profitable au spécialiste de la littérature. Ce phénomène existait par ailleurs à l'époque où j'étais étudiant. Dans les années 1970 cette ouverture pluridisciplinaire était une obligation : on lisait Roman Jakobson, Roland Barthes, Michel Foucault... Depuis la fin des années 1990 l'Université a vécu un repli disciplinaire impressionnant dû à l'impératif de produire de plus en plus d'articles pour avancer dans la carrière et de se soumettre à l'évaluation de spécialistes qui ne valorisent que ce qu'ils considèrent comme pertinent. L'interdisciplinarité est pénalisée. De la même façon, la pression croissante à publier, la valorisation des carrières sur la base de la quantité de publications, enlève le temps d'inventer. La formulation d'un projet est aujourd'hui soumise à un système qui oblige à s'aligner sur des instructions dictées d'en haut et qui cloisonnent rigidement les disciplines. Cela comporte le risque que l'Université ne produise plus d'intellectuels réactifs et engagés. Et cette difficulté frappe surtout les jeunes qui, entre 20 et 40 ans - cette période de leur vie où les idées devraient foisonner -, n'ont pas le temps de réfléchir 
par manque de temps et parce qu'ils ont les mains liées par le système. Il faut encourager l'ouverture...

... QUI PASSE AUSSI PAR UN DIALOgUe INTERNATIONAL, DANS LE CADRE D'UNE
COMMUNAUTÉ QUI ÉCHANgE AU-DELÀ DES FRONTIÈres. CE DIALOgUE DOIT-IL À VOTRE AVIS
SE FAIRE DANS UNE LINGUA FRANCA, L'ANgLAIS, AU RISQUE DE SIMPLIfIER NOTRE
RAISONNEMENT, D'EN APLATIR LES SUBTILITÉS, OU DANS UN CONTEXTE DE
PLURILINGUISME?

Choisir l'anglais pour tous, dans nos disciplines, est une grave erreur. Si pour les scientifiques la langue est un instrument pour parler de choses qui sont extérieures au langage, dans notre cas la langue est tout, car sans celle-ci nos disciplines n'existeraient pas. Accepter l'anglais comporte aussi l'adhésion à une série de stéréotypes et de modes de pensée qui appartiennent à cette langue et à cette culture, car chaque langue a les siens propres. Cela revient donc à dénaturer la pensée. Je pense toujours au monde gréco-romain et au monde des XVII $-\mathrm{XVIII}^{\mathrm{e}}$ siècles où les intellectuels voyageaient à travers l'Europe et se comprenaient, car ils n'avaient pas l'angoisse que nous, post-romantiques, avons développée, de la langue comme quelque chose d'inaccessible, d'extraordinaire, de merveilleux... On devrait aller audelà de la traduction, vers un plurilinguisme qui nous permette de nous comprendre même en parlant des langues différentes, chacun utilisant la sienne, tout en allant vers l'autre. Il s'agit de faire un effort réciproque, en favorisant une maitrise décente des quelques langues qui nous permettent de lire dans un idiome différent du nôtre. Quand on écrit en revanche, c'est l'acte d'écrire lui-même qui est créatif. Comme le dit Jules Renard: "L'inspiration, ce n'est peut-être que la joie d'écrire: elle ne la précède pas. » En écrivant, arrivent les idées. Et cela n'est pas possible en se forçant à écrire dans une langue qui n'est pas la sienne. Il faut maintenir les langues vivantes. Il est tout à fait possible de se comprendre, surtout si l'on a un bon motif pour le faire.

Notre avenir devra abandonner l'idée d'une langue commune à tous ainsi que l'obsession de la fidélité dans la traduction, contrainte qui naît avec la traduction des textes sacrés, la Septante et ensuite saint Jérôme et qui est en revanche étrangère à d'autres horizons, comme les cultures chinoise, indienne et arabe ${ }^{3}$. Cette anxiété de l'authenticité est typiquement occidentale et postchrétienne. Je souhaite que l'on arrive à se libérer de cette angoisse. Cela donnerait une grande impulsion à l'échange.

DANS CETTE BELLE PROJECTION VERS L'AVENIR PLUS OU MOINS IMMÉDIAT, POURRIEZ-VOUS NOUS PARLER DE VOS TRAVAUX EN CHANTIER?

Je suis en train de terminer un petit livre d'engagement civil qui concerne la «tenaille » de l'identité. J'ai l'impression que nous sommes de plus en plus contraints dans une opposition binaire entre le «nous » et l'" autre ». Cela se manifeste dans la sacralisation de notre identité : c'est ce que fait Matteo Salvini en tenant un chapelet dans la main ; il ne récite pas le chapelet, il le brandit. C'est l'objet sacralisant qui lui permet de faire ses discours sur l'identité. De même pour d'autres phénomènes typiques de notre culture qui sont liés à la fois à un désir et à une peur de s'identifier. Dans mon livre en chantier, je consacre un chapitre au mythe du «grand remplacement ", cette théorie complotiste d'extrême droite, raciste et xénophobe, récemment popularisée par Renaud Camus, selon laquelle il existerait un plan délibéré de substitution de la population française et européenne par une population non européenne, originaire en premier lieu d'Afrique noire et du Maghreb. J'analyse 
cette théorie en utilisant les instruments de la culture ancienne et tout particulièrement le concept romain de fama : comment fonctionne-t-elle ? Comment s'affirme une rumeur? selon quels mécanismes? Je m'appuie sur l'Antiquité classique pour comprendre une rumeur moderne.

Sur le plan des études dans le domaine plus spécifiquement ancien, je continue à travailler sur la question de la parole parlée à Rome et sur les modalités avec lesquelles la culture romaine s'est formée à partir non pas de la parole écrite mais de la parole parlée, ce qui se manifeste dans plusieurs domaines qui vont du droit à la composition littéraire. Je m'arrête aussi sur certaines notions clés de la culture romaine, comme le mot fatum, « ce qui est dit », " la parole », mais aussi le « destin », alors que pour les Grecs le « destin » est un « morceau » (moira). Cela montre, encore une fois, l'importance des mots pour entrer dans l'imaginaire.

\section{NOTES}

1. Réponse qu'on peut trouver dans une autre monographie récente de M. Bettini, Homo sum. Essere «umani» nel mondo antico (Turin, Einaudi, 2019, traduit en français par M. Rousset Grenon : Contre les barbares, Paris Flammarion, 2020), où le débat actuel sur l'accueil des migrants et sur les droits de l'homme s'enrichit, en partant de quelques vers de l'Énéide, d'une réflexion sur comment les Anciens pensaient le ius humanum avant la Déclaration des droits de l'homme de 1948. Le but n'est pas de retrouver de prétendues racines de l'humanisme moderne, mais plutôt de mesurer l'altérité des Anciens, les écarts qui nous opposent, et de comprendre comment les Grecs et les Romains se sont confrontés à ces thèmes, entre fermeture (cf. les Athéniens et leur «mythe » de l'autochthonie) et ouverture et hybridation (la nature agrégative de l'identité romaine).

2. Il s'agit du livre Il grande racconto dei miti classici, Bologne, Il Mulino, 2015.

3. Cf. à ce propos M. Bettini, Vertere. Un'antropologia della traduzione nella cultura antica, Turin, Einaudi, 2012. 